\title{
Nonocclusive, Clean Permanent Right Atrial Catheter Dressing Change Procedures Compared With Occlusive, Sterile Permanent Right Atrial Catheter Dressing Change Procedures in Children With Cancer
}

\author{
Sally K. Hutchinson, BSN, PNP, \\ Mary Waskerwitz, RN, BSN, CPNP, Kathleen Martin, RN, \\ Walter Faubion, RN, MS, and Susan Revesz, RN, BSN
}

Nursing practices at many institutions advocate the use of occlusive, sterile permanent right atrial catheter (PRAC) dressing change procedures. Few of these practices are based on research data. However, nursing literature suggests that employing simpler procedures or no dressing at all may be acceptable.

Using a randomized, experimental, crossover study design, a comparison was made between an occlusive, sterile PRAC (standard) dressing change procedure and a nonocclusive clean PRAC (experimental) dressing change procedure in children with cancer. The objectives of this study were to compare infectious complication rates, differences in cost, and patient satisfaction associated with each procedure. Thirty-eight patients were entered on the study; 24 were evaluable. Observations of the exit site condition were made with each dressing change and recorded on data capture forms by a parent (or primary nurse if hospitalized). Evaluations were also made by the primary nurse during routine visits to the outpatient department. Patients and parents performed the standard and experimental dressing change procedures, in random sequence, for approximately 3 months each. Parents provided demographic data at registration and indicated dressing change preference in an endof-study questionnaire. Initial analysis was performed using McNemers $\chi^{2}$ formula for matched pairs.

The results of our study suggest that there is no significant difference in infectious complications at the exit site between sterile, occlusive PRAC dressing change procedures and nonocclusive, clean PRAC dressing change procedures in children with cancer. In addition, the nonocclusive dressing change procedure is less costly and easier to perform. Our study is ongoing with a goal of accumulating data on 50 evaluable patients.

Studies such as this have a significant impact on pediatric oncology nursing because they help to define patient care practices that best serve patients and their families in a safe, yet practical manner.

From the University of Michigan Medical Center, Ann Avbor, MI. (1) 1990 by Association of Pediatric Oncology Nurses. 